\title{
STORIA DELLA SCRITTURA E STORIA DELLA SOCIETÀ
}

"Il est légitime, à certains moments de la formation d'une science, de passer en revue les matériaux ressemblés, les premiers résultats obtenus, surtout por tracer les linéaments des recherches prochaines».

(M. Cohen, Matériaux pour une sociologie du langage, I, Paris 1971, p. 6).

1. Nell'ambito della storia delle scritture, e in particolare della storia della scrittura latina, esiste da tempo un filone di ricerca che tende a porre in rilievo e a fare oggetto della propria indagine $\mathrm{i}$ rapporti intercorrenti in diverse situazioni storiche fra i sistemi di scrittura, le forme grafiche e i processi di produzione di testimonianze scritte da un lato, e le strutture socio-economiche delle società che elaborano, adoperano e manipolano tali prodotti culturali dall'altro.

Fino a qualche tempo fa questo filone di ricerca è rimasto praticamente estraneo allo sviluppo degli studi paleografici, sul quale ha influito assai scarsamente; innanzi tutto perchè gli studiosi che lo hanno più o meno occasionalmente perseguito non hanno adottato metodologie di ricerca e di analisi comuni; quindi perchè tali studiosi in molti casi non erano propriamente paleografi, o non lo erano "a tempo pieno», come usa dirsi; e infine perchè, anche quando si trattava di paleografi «riconosciuti», essi appartenevano ad aree culturali marginali o comunque deboli della tradizione storiografica europea, cosicchè $i$ loro contributi erano destinati in partenza a cadere nel vuoto, senza che gli storici della scrittura -ovvero i paleografi di altre e più prestigiose aree e tradizioni- ne prendessero in considerazione le proposte metodologiche e problematiche.

Questo contributo nasce sotto gli auspici di una citazione eloquente di un grande linguista francese di formazione coerentemente marxista, Marcel Cohen (+1974); è giusto dunque iniziare proprio da lui la nostra rassegna dei maggiori contributi finora apportati ad una prospettiva di tipo sociale degli studi di storia della scrittura.

Tale prospettiva era insita già in un volumetto, intitolato L'Écriture ', che il Cohen pubblicò nel 1953 e che costituiva un riassunto anticipato della più

' M. COHEN, L'écriture, Paris, 1953. 
grande -e celebre- opera che sarebbe seguita cinque anni appresso: La grande invention de l'écriture", ove all'inizio si può leggere una massima cui l'intera trattazione è in pratica uniformata: «L'emploi de l'écriture est fonction de son utilité dans une société donnée» '. Questa impostazione generale era stata colta con acutezza da un linguista sovietico, V. A. Istrin, il quale, recensendo nel 1955 il primo contributo del Cohen, osservava che «les besoins sociaux d'écriture occupent une place tout à fait particulière parmi ces facteurs" [di sviluppo della scrittura]; e ancora; «le développement des besoins sociaux d'écriture, lié étroitement au développement de toute la société, conditionne les lois historiques du développement de l'écriture» ${ }^{4}$. L'Istrin, in realtà, andava evidentemente oltre le più prudenti enunciazioni del linguista francese. nello stabilire un rapporto immediato e diretto di dipendenza fra quelli che egli definiva «i bisogni sociali di scrittura» e le cosiddette "leggi storiche di sviluppo della scrittura" stessa '. Ma certo è che Cohen stesso tradusse e introdusse con evidente compiacimento il brano dell'Istrin all' interno della sua opera maggiore, dal che si può dedurre senza tema di errare che egli finì per condividerne il giudizio.

Certo è che nella più recente produzione della paleografia latina sovietica questo tipo di impostazione appare minoritario o addirittura assente. In una rassegna dei lavori di paleografi latini suoi compatrioti apparsi negli anni 1960-1966, pubblicata nel 1967, Anna Lublinskaya, la più autorevole rappresentante di una tradizione di studi risalente ad Olga Dobiache Rojdentsvenskaya, rivendicava una metodologia di carattere puramente tecnico e l'acquisizione indiscussa dell'impostazione della cosidetta «scuola francese» di paleografia: «Les paléographes soviétiques apprécient vivement la méthode d'analyse paléographique introduite par $\mathrm{M}$. Jean Mallon et par d'autres paléographes, l'estimant très fructueuse. Nous l'appliquons dans nos travaux de recherche»".

2. Eppure proprio da alcuni paesi dell'Est europeo, ove l'unica storiografia ammessa era quella di ortodossa impostazione marxista, sono venuti, soprattutto nel corso degli anni Cinquanta, in singolare coincidenza con gli studi maggiori di Marcel Cohen, alcuni contributi di studiosi che hanno in

2 M. COHEN, La grande invention de l'écriture et son évolution, I-II, Paris 1958.

ibid., I, p. 7.

4 cit. ibid. (in traduzione francese), II, p. 24.

s Sugli orientamenti ideologici e metodologici della linguistica sovietica negli anni Cinquanta e sul collegamento più o meno meccanico da essa operato fra uso della lingua e strutture socio-economiche, cf. le osservazioni critiche dello stesso COHEN in Pour une sociologie du langage, Paris 1956, pp. 29-32.

'A. LUBLINSKAYA, Les travaux des savants soviétiques en paléographie latine (19601966), in «Scriptorium», XXI (1967), pp. 100-103. 
qualche misura impostato in modo del tutto nuovo la metodologia della ricerca storico-grafica. Mi riferisco in particolare agli studi paleografici dell'ungherese Istvan Hajnal e del grande storico polacco Aleksander Gieysztor.

L'opera più nota in Europa occidentale dello Hajnal è la sua monografia dedicata alla storia dell' insegnamento della scrittura nelle università medievali, la cui seconda edizione fu pubblicata, per cura di Lazlo Mezey, nel 1959 '. Si tratta, com'è ben noto, di un grande affresco storico-culturale, che si risolve in una suggestiva ricostruzione dei metodi di insegnamento e di educazione grafica propri all'Europa (e in particolare all'Europa centro-orientale) tardomedievale e in un importante contributo alla storia delle professioni intellettuali. L'opera non ha mancato di suscitare perplessità e riserve; e sul piano propriamente paleografico le ipotesi dello Hajnal sembrano attualmente nel loro complesso insostenibili. Ciò non toglie che uno dei pregi maggiori della ricerca del paleografo ungherese appaia ancora oggi il solido e consapevole ancoraggio dei fenomeni grafico-culturali al moto generale di evoluzione di una società vista nel suo complesso, che la ispira: «L'écriture -egli affermava- tout comme les autres formes de civilisation, est un moyen né de l'ensemble de la société: son avenir depend du caractère systématique de sa pénétration dans la société» "; e ancora: "Ce n'est pas de l'écriture que l'écriture a évolué toujours plus avant, tout comme aucun autre moyen technique ne se développe à partir du moyen précédent, mais par la reciprocité continuelle avec la société»".

Per il Gieysztor basterà rinviare al suo antico articolo (del 1952-53) sulle origini della minuscola carolina, che sottolineava con forza il legame fra il nuovo tipo di scrittura e le necessità organizzative e comunicative dell'aristocrazia carolingia ${ }^{10}$, e al suo relativamente recente manuale di paleografia latina".

Il programma di ricerca ideologico e metodologico dello Hajnal fu reso esplicito da un contributo di un giovane storico rumeno, Sigismund Jakó, presentato nel corso del X Congresso internazionale di scienze storiche svoltosi a Roma nel 1955, che recava un significativo sottotitolo: Considerations sur

'L'enseignement de l'écriture aux Universités médiévales, 2a ed., a c. di L. MEZEY, Budapest 1959.

8 ibid., p. 9.

9 ibid., p. 31.

11 Problem Karolínskiej Reformy Pisma, in «Archeologij», V (1952-52), pp. 155-77 (con riassunto in francese); e anche A. PRATESI, Le ambizioni di una cultura unitaria: la riforma della scrittura, in Nascita dell'Europa e Europa carolingia: un'equazione da verificare, I, Spoleto 1981, pp. 507-23.

$"$ Zaris dziejów Pisma tacinskiego, Warszawa 1973; ma anche il saggio Certains problèmes de l'évolution de l'écriture romaine, in "Archeologia polona», XIV (1973), pp. 179. 87. 
l'étude de la paléographie sur de nouvelles bases ${ }^{12}$. Dopo aver sottolineato la necessità per la paleografia di acquisire "un rôle indépendent et important dans le domaine de l'éclaircessement des rapports de la société avec la culture», il Jakó affermava che «cette discipline nouvelle -cioè la paleografia fondata su nuove basi- doit s'occuper -en dehors de l'écriture et du corps des préposés que en étaient chargés, ainsi que de l'histoire de toutes les couches sociales connaissant son usage, donc de son rôle social»; in tal modo la paleografia si trasformerebbe da «une discipline empirique de l'écriture, telle qu'elle a été conçue jusqu'à présent, en une histoire de l'écriture dans l'acception la plus large du termen".

E'interessante rilevare oggi, a trent'anni di distanza, che questa sẹrie di testi, insieme con le provocazioni e le nuove prospettive di metodo in essi contenute, caddero praticamente nel vuoto, e che il piccolo mondo della paleografia latina o quello, più vasto, degli storici della scrittura, non seppero nè riceverne, nè decifrarne il messaggio. Alcune delle ragioni di tale rifiuto risiedono certamente nella debolezza oggettiva delle ricerche specifiche cui il messaggio stesso si accompagnava e nella estraneità di tali ricerche, e dei relativi proponenti, a quel metodo formale di analisi del prodotto manoscritto che in Occidente proprio allora si andava affermando fra i paleografi latini per merito della «scuola francese»; ma certamente un grande -e forse decisivoruolo fu giocato dalla diffidenza ideologica, oltre che dalla carenza di informazione e dalla pigrizia culturale che caratterizzano in genere tutte le tradizioni disciplinari relativamente piccole, istituzionalizzate e fortemente segnate da un sapere tecnico.

3. Nel corso degli anni Sessanta la prospettiva di studi che individuava nel rapporto fra scrittura e società un nodo essenziale della storiografia contemporanea ricevette nuovo impulso dalle prospettive aperte dagli studi di storia dell' alfabetismo proposti da due importanti storici operanti in area anglosassone: Carlo Maria Cipolla e Lawrence Stone.

Al Cipolla si deve fra l'altro una vivace ed intelligente sintesi di storia dell'alfabetismo nel mondo occidentale pubblicata per la prima volta in Inghilterra nel 1969 in una collana di divulgazione ${ }^{14} \mathrm{e}$ tradotta in italiano (con poca fortuna) nel $1971^{\text {'s }}$. Si tratta di un saggio fondato su una prospettiva illuministicamente progressista delle sorti dell' umanità, nell'ambito della qua-

12 S. JAKÓ, Les débuts de l'écriture dans les couches läques de la société féodale en Transylvanie, in "Nouvelles études d'histoire», présentées au X' Congrès des Sciences Historiques, Rome 1955, pp. 209-23.

13 ibid., pp. 209-10.

14 C. M. Cipolla, Literacy and development in the West, London 1969.

1s C. M. CIPOLLA, Istruzione e sviluppo. Il declino dell'analfabetismo nel mondo occidentale, Torino 1971. 
le sviluppo economico e sviluppo delle capacità culturali sono visti come elementi necessariamente complementari. Allo Stone, oltre a molti e notissimi studi sulla società inglese di antico regime, si devono in particolare due articoli di grande novità e rilievo pubblicati rispettivamente nel 1964 e nel 1969 in $P$ ast and Present e riguardanti il primo la «rivoluzione educativa» verificatasi in Inghilterra fra Cinque e Seicento e l'altro il rapporto fra educazione e alfabetismo in Inghilterra fra l'età di Cromwell e questo secolo ${ }^{16}$.

E' impossibile -e sarebbe comunque inopportuno- tentare di riassumere in questa sede gli spunti e i dati forniti dallo Stone ai fini di una valutazione del rapporto fra strutture sociali e diffusione dell' alfabetismo nell'Europa moderna. Ma vanno almeno rilevate alcune affermazioni dello storico inglese di grande importanza metodologica: 1) che fra condizione sociale dei singoli e capacità di scrivere e di leggere esiste una strettissima connessione ${ }^{17}$;2) che fra capacità di leggere e capacità di scrivere è esistita per secoli una forte differenziazione ${ }^{18}$; 3) che lo sviluppo economico può essere causa di un abbassamento, e non di un aumento, del tasso di alfabetismo delle popolazioni interessate ${ }^{19}$; 4) che l'alto sviluppo demografico contrasta e non favorisce una diffusione di massa dell'alfabetismo ${ }^{20}$.

Allo Stone si deve anche la individuazione di cinque diversi livelli di capacità grafico-culturali corrispondenti, nell'ambito del periodo e della società studiati, ad altrettante stratificazioni sociali: 1) scrittura del solo nome e scarsa lettura $=$ poveri; 2 ) capacità di leggere, scrivere a far di conto $=$ classi medio-basse (artigiani, piccoli commercianti, operai qualificati, ecc.); 3) livello di istruzione secondaria, capacità amministrativa = classi medie (professioni $\mathrm{e}$ affari); 4) educazione sui classici come preparazione agli studi universitari = classi superiori; 5) università = professionisti e dilettanti delle classi superio$\mathrm{ri}^{21}$. E infine sempre allo Stone dobbiamo una chiara e non equivoca definizione del rapporto intercorrente nei secoli -e in ogni società alfabetizzata- fra scrittura e pubblici poteri: «E' perfettamente vero che per millenni uno dei principali (anzi, con la trasmissione dei testi sacri, il principale) usi della scrittura fu rivolto a rafforzare il controllo burocratico e il potere militare...." ${ }^{22}$.

16 Mi riferisco a The Educational Revolution in England. 1560-1640, in Past and Present, 28 (1964, luglio), pp. 41-80 e a Literacy and Education in England. 1640-1900, ibid., 42 (1969, febbr.), pp. 69-139.

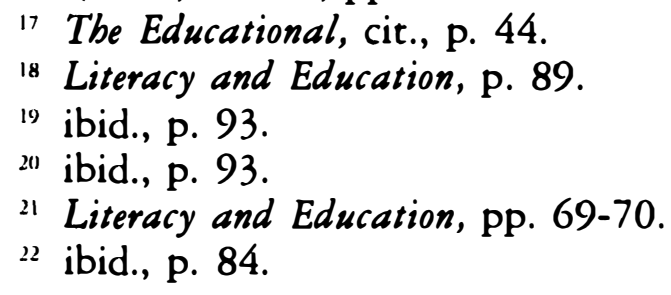


Agli studi dello Stone seguirono nel mondo anglosassone quelli di molti altri storici della cultura nell'età moderna tesi ad illustrare, a volte con metodi di tipo quantitativo, momenti particolari del processo di diffusione dell'alfabetismo: que basterà ricordare soltanto, per l'Inghilterra, il dibattito sul rapporto fra prima rivoluzione industriale e alfabetismo, svoltosi su Past and Present ${ }^{23}$, e le opere di Cressy ${ }^{24}$ e, per il medioevo, di Clanchy ${ }^{25}$; per gli Stati Uniti i contributi, anche bibliografici, di Graff ${ }^{26}$; d'altra parte non si può ometere il ricordo dell'opera complessiva di F. Furet e J. Ozouf sulla storia dell'alfabetismo nella Francia moderna ${ }^{27}$. A noi, in questa sede, importa soprattutto rilevare che nessun elemento, o stimolo, o suggestione di questo complesso travaglio storiografico intorno alla diffusione e all'uso sociale della scrittura è stato accolto, neppure di riflesso, all'interno della paleografia: di questi temi non si è mai parlato in sede di Comité International de Paléographie ${ }^{28}$; i nomi di Cipolla, di Stone, di Clanchy non figurano nelle bibliografie paleografiche più recenti ${ }^{29}$ e neppure nell'ultima sintesi di paleografia latina dovuta a Bernhard Bischoff e tradotta (o in via di traduzione) in più lingue ${ }^{30}$.

$\mathrm{Ma}$ in questi casi, oltre ai motivi enunciati più sopra, bisogna ricorrere, per spiegare le ragioni delle omissioni e dei silenzi, non tanto, o non soltanto,

${ }^{23}$ Un riassunto della questione con le relative indicazioni bibliografiche in A. PETRUCCI, Per la storia dell'alfabetismo. Metodi, materiali, quesiti, in "Quaderni storici», 38 (1978), pp. 463-5.

${ }_{24}$ D. CRESSY, Literacy and Social Order. Reading and Writing in Tudor and Stuart England, Cambridge 1980.

${ }^{25}$ M. T. ClanCHY, From Memory to Written Record. England 1066-1307, London 1979.

26 Di cui qui ricorderò, fra i molti contributi sull'argomento, soltanto The Literacy Myth. Literacy and Social Structure in the Nineteenth Century City, New York-San FranciscoLondon 1979.

27 F. FURET-J. OzOUF, Lire et écrire. L'alphabétisation des Français de Calvin à Jules Ferry, I-II, Paris 1977.

${ }_{28}$ Come risulta, ad esempio, dagli atti della riunione del Comité svoltasi a Monaco di Baviera nel 1981: Paläographie 1981. Colloqium des Comité international de Paléographie. Referate, a cura di G. SILAGI, München 1982.

${ }^{29}$ Come in quella, pur ampia, precisa, informata di L. BOYLE, Medieval Latin Palaeography. A Bibliographical Introduction, Toronto 1984 (estesa peraltro cronologicamente soltanto fino all'età rinascimentale).

30 B. BISCHOFF, Paläographie des römischen Altertums und des abendländiscen Mittelalters, Berlin 1979; ora anche in traduzione francese Paléograpbie de l'antìquité romaine et du moyen âge occidental, Paris 1985.

Si noti che già nel 1978 A. BARTOLI LANGELI (Ancora su Paleografia e storia della scrittura, in Scrittura e Civiltà, II [1978], p. 278, núm. 7) lamentava la sordità dei paleografi italiani a tali nuove prospettive. 
alla diffidenza ideologica, quanto piuttosto alla tradizionale impostazione filologica e medievistica, che connota ancora tanti studiosi, e tanti studi, di paleografia latina e greca.

4. Fra gli anni Sessanta e gli anni Settanta una forte spinta al rinnovamento degli studi paleografici nel senso di un più diretto e puntuale collegamento fra gli eventi e $i$ prodotti grafici da una parte e la società nel suo complesso dall'altra è venuta da una serie di studi nati o promossi nell' ambiente di ricerca romano.

In un saggio apparso nel $1969{ }^{31} \mathrm{mi}$ avveniva di indicare come questioni di grande importanza per la storia della scrittura (di ogni scrittura) quelle attinenti alla diffusione sociale delle capacità di scrivere nell' ambito di ciascuna organizzazione sociale e alle funzioni, in genere molteplici, che all'interno di ciascuna società la scrittura svolge: le questioni, insomma, del chi scriveva o del perchè si scriveva ${ }^{32}$. Ne discendeva un orientamento delle ricerche volto più all' individuazione e allo studio delle testimonianze grafiche e degli scriventi che non delle singole tipologie grafiche in sè e per sè; anche se si confermava l'autonomia e l'imprescindibilità del metodo paleografico di analisi formale delle singole testimonianze scritte. Tale orientamenti travarono conferma in una relazione tenuta nel 1971 alla XIX Settimana di studi altomedievali di Spoleto, dal titolo Libro, scrittura e scuola ${ }^{33}$, e in un saggio teorico e metodologico edito soltanto nel 1979 , ma radatto nel $1973^{\text {i4 }}$, oltre che in contributi successivi di diversa ampiezza e rilievo ${ }^{3}$.

Mi pare che oggi si possa affermare che da interventi di tale tipo -dovuti non soltanto a chi scrive, ma anche ad altri studiosi- la paleografia viene individuata come una disciplina storica che tende alla ricostruzione dei metodi e delle condizioni di produzione delle testimonianze scritte e dell'uso e della diffusione delle capacità di scrivere nelle diverse situazioni storico-sociali; e ciò secondo un orientamento che, almeno per quanto riguarda chi scrive, può essere definito più o meno indirettamente di indirizzo marxiano, in quanto fondato sul presupposto che sia appunto la strutturazione di ciascuna società organizzata a determinare o ad influenzare in qualche misura gli usi e le funzioni della scrittura o delle scritture adoperate nel suo ambito e per

"Scrittura e libro nell'Italia altomedievale. Il sesto secolo, in "Studi medievali», $3^{2}$ serie, X (1969), pp. 157-213.

3 Ibid., pp. 157-9.

33. In La scuola nell'Occidente latino nell'alto medioevo, Spoleto 1972, pp. 313-37.

${ }^{34}$ Funzione della scrittura e terminologia paleografica, in Palaeographica, Diplomatica et Archivistica. Studi in onore di Giulio Battelli, I, Roma 1979, pp. 3-30.

is Cf. per questo A. PETRUCCI, Scrittura, alfabetismo e produzione libraria nell'alto medioevo, in La cultura in Italia fra tardo antico e alto medioevo, Roma 1981, II, pp. 53851. 
conseguenza anche il diverso atteggiarsi e modificarsi delle singole tipologie grafiche.

Tale orientamento, e la spinta conseguente ad un allargamento qualitativo e cronologico del territorio degli studi paleografici, hanno provocato verso la fine degli anni Settanta in importante incontro interdisciplinare svoltosi a Perugia "e la nascita di una serie di studi di storia dell' alfabetismo e dell'educazione grafica basati prevalentemente su analisi paleografiche di testimonianze scritte spontanee vergate in scritture «usuali» o «elementari di base», che è lungi dall'essere esaurita e che ha portato a qualche risultato di rilievo anche nel campo più specifico e limitato della storia delle tipologie scrittorie ".

In effetti la metodologia di ricerca che sottostà a questo tipo di studi è basata in prima istanza sulla puntuale (e tradizionale, oserei dire) analisi formale-grafica delle testimonianze scritte prese in esame, per arrivare quindi ad individuare una serie di elementi storico-grafici legati fra loro da stretta connessione analogica; i quali elementi, innanzi tutto, corrispondono a diversi aspetti dell'uso (individuale o sociale) della scrittura nella situazione che si studia; quindi, una volta individuati, analizzati e posti fra loro a confronto, finiscono per rappresentare i diversi momenti della conoscenza storico-grafica che ciascuna volta si tenta, appunto attraverso questo metodo di analisi, di costruire.

Tali elementi, da individuare e da analizzare nel corso della ricerca, sono, in successione, i seguenti:

1) la tipologia grafica di base cui ciascuna testimonianza scritta presa in esame si rifa e che, secondo una definizione di Robert Marichal, può essere considerata il suo "pôle d'attraction" ${ }^{38}$, o, con definizione di Franco Bartoloni, la «categoria largamente comprensiva» di riferimento ${ }^{39}$;

16 I cui atti sono stati pubblicati sotto il titolo Alfabetismo e cultura scritta nella storia della società italiana, Perugia 1978, e, in modo non completo, in "Quaderni Storici», 38 (1978), pp. 437-700. Sull'iniziativa, cf. A. BARTOLI LANGELI, Ancora su paleografia e storia della scrittura, cit., pp. 275-94; e la replica di A. PRATESI, Paleografia in crisi?, ibid., III (1979), pp. 329-37.

1) Si vedano, oltre i saggi citati più avanti alla nota 42, anche A. FRASCADORE, Un'indagine su alfabetismo e cultura scritta: S. Pietro di Galatina alla fine del '500, in "Scrittura e Civiltà», 5 (1981), pp. 199-229; A. BARTOLI LANGELI, Culture grafiche e competenze testuali nel Quattrocento italiano (la prima matricola della confraternita del $S$. Anello di Perugia, 1487-1542), in Retorica e classi sociali, a cura di M. CORTELAZZO, Padova 1983, pp. 83-94; F. GIMENO BlAY, La escritura en la diócesis de Segorbe. Una aproximación al estudio del Alfabetismo y la Cultura Escrita en el Alto Palancia (1383-1458), Valencia 1984 (Universidad de Valencia, Departamento de Paleografía Diplomática, «Estudios», 4).

"In un rapporto dal titolo Paléographie latine et française, in "Annuaire», École pratique des Hautes Études, 1964-65. p. 230.

3) In La nomenclatura delle scritture documentarie, in «Relazioni», X Congresso 
2) il grado di corsività di esecuzione dell'esempio (posato, semicorsivo, corsivo, corrente);

3) il grado di abilità esecutiva del singolo scrivente così come esso è rivelato dalla testimonianza da lui redatta, che può essere scritta in "elementare di base», in scrittura "usuale» o in tipologia grafica vicina al canone ( «pura») o tipica di determinate categorie di professionisti dello scritto («professionale») "

4) il livello di educazione grafica e di cultura scrittoria dello scrivente stesso, che è dedotto principalmente dall'analisi di quanto esposto al punto precedente; chi scrive in "elementare di base», infatti, difficilmente è andato oltre i primi gradini dell'insegnamento dello scrivere; chi scrive in "usuale», in particolare se lo fa con fluidità e scioltezza, ha certamente ricevuto un notevole complesso di cognizioni grafiche, che la pratica ha mantenuto in esercizio e a volte accresciuto; chi scrive in tipologie grafiche corrispondenti al canone o in scritture "professionali» può essere considerato un vero e proprio professionista della penna. Inoltre lo studio del livello di educazione grafica e di cultura scrittoria dei singoli scriventi si giova moltissimo dell' analisi, anchessa necessaria, di numerosi altri dati grafici ed extragrafici, quali l'uso e la frequenza di abbreviazioni, l'uso e la frequenza di segni di punteggiatura e di sistemi di impaginazione e di articolazione del testo, l'uso di maiuscole (e di quali maiuscole), l'uso di segni tecnici (per indicare la monetazione o altro) e così via;

5) il confronto fra le diverse testimonianze grafiche costituenti la documentazione indagata e perciò fra le varie personalità di scriventi individuate fornisce, a conclusione dell' indagine, e per ciascuna concreta situazione storicografica presa in esame, il quadro complessivo delle tipologie grafiche in uso, dell'educazione grafica e dell'uso sociale di scrittura, della presenza e del ruolo di determinate fasce di scriventi abili o di professionisti dello scritto; con tutte le conseguenze che se possono trarre ai livelli più diversi.

5. Una prospettiva di studi caratterizzata, nelle problematiche e nei metodi, nel modo indicato nel precedente paragrafo, può essere adoperata per studiare in modo utile e nuovo situazioni e problematiche particolari all'interno della storia della scrittura e delle testimonianze scritte finora mai poste come possibile terreno di indagine o soltanto accennate o sfiorate in contesti storio-

internazionale di scienze storiche. I. Metodologia. Problemi generali. Scienze ausiliarie della storia, Firenze 1955, p. 440.

40 Su tali gradazioni di capacità esecutive cf. il già ricordato contributo Scrittura, alfabetismo ed educazione grafica, pp. 167-8, e le proposte di A. BARTOLI LANGELI, Sulla classificazione formale delle testimonianze grafiche uspontanee» (a proposito del modello elaborato da Jean Queniart), in Alfabetismo e cultura scritta. Seminario permanente, «Notizie», dicembre 1980, pp. 31-6. 
grafici fra loro lontani e non comunicanti. Ma in realtà lo studio del ruolo sociale della scrittura, con tutto ciò che esso comporta, va bene al di là delle limitate tematiche legate all'uso della scrittura da parte di scriventi non professionali e alla storia dell'alfabetismo cui finora si è accennato. Esso investe direttamente -e proficuamente- la storia del libro manoscritto, sia dal punto di vista della produzione che da quello dell'uso, la storia delle scritture d'apparato e di quelle esposte (cioè l'epigrafia), la storia delle tecniche e delle materie scrittorie, e in pratica ogni aspetto che riguardi la produzione, la diffusione e l'uso di testimonianza scritte. ${ }^{41}$

A scopo puramente esemplificativo indicherò qui di seguito alcuni fra $\mathrm{i}$ molti temi che si sono prestati finora o che comunque si prestano ad indagini quali quelle fin qui prospettate e che riguardano sia l'età medievale sia quella moderna; ma, com' è evidente, la esemplificazione potrebbe facilmente essere assai più vasta ed articolata.

Per quanto riguarda l'alto medioevo europeo l'uso limitato della capacità di scrivere da parte di alcune categorie di alfabeti laici ed ecclesiastici in situazioni di analfabetismo sociale diffuso constituisce un tema di ricerca che può giovarsi di un materiale abbondante e facilmente accessibile, costituito dalle sottoscrizioni autografe ai documenti e che è suscettibile di arrecare dati e rilievi di notevole interesse per quanto riguarda lo sviluppo delle tipologie grafiche in uso ${ }^{42}$.

Per quanto riguarda il tardo medioevo italiano un fenomeno che appare sempre più di grande interesse non soltanto agli occhi degli storici del libro, ma anche ai filologi romanzi e italiani e più in generale a tutti gli storici delle tradizioni volgari è quello costituito dalla progressiva discesa del libro come strumento di cultura scritta all'interno degli strati medio-bassi della società europea dei secoli XIII-XV, in corrispondenza con una notevole crescita progressiva dell'alfabetismo urbano; fenomeno che è caratterizzato da due diversi aspetti complementari e non separabili: l'uno che riguarda la produzione di libri con testi volgari e con tipologia grafico-materiale propria all'interno di ben precisati ambienti borghesi e popolari; e l'altro che riguarda l'uso, la circolazione e il possesso di tali libri all'interno di questi stessi ambienti, che peraltro

4l Per le prospettive di una "sociologia della scrittura», si vedano gli spunti di G. R. CARDONA, Antropologia della scrittura, Torino 1981, pp. 89-131 (ma anche nell' Introduzione le pp.7-10).

${ }^{42}$ Cf. per questo alcuni studi recenti: A. PETRUCCI, Libro, scrittura e scuola, in La scuola nell Occidente latino dell'alto medio evo, Spoleto 1972, Pp. 313-37; G. G. FISSORE, Cultura grafica e scuola in Asti nei secoli IX e X, in "Bullettino dell'Istituto storico italiano", 85 (1974-75), pp. 17-51; A. PETRUCCI-C. ROMEO, Scrittura e alfabetismo nella Salerno del IX secolo, in «Scrittura e Civiltà», 7 (1983), pp. 51-112. 
costituivano anche il terreno di cultura privilegiato per la creazione di più $o$ meno estesi testi di carattere memoriale e storico ${ }^{43}$.

Più direttamente e strettamente legato alla tematica del rapporto fra potere pubblico e scrittura appare lo studio delle scritture d'apparato esposte in luoghi pubblici, che può portare all' individuazione di grandi e appariscenti "programmi di esposizione grafica», come quello organizzato a Roma per conto di Sisto $\mathrm{V}$ dal calligrafo Luca Orfei, e comunque in ogni caso ad una più attenta e vigile considerazione del valore sociale, dal punto di vista culturale e dal punto di vista politico, della presenza pubblica dello scritto esposto in forme solenni e organizzate ${ }^{44}$. A tale tipo di indagini non può d'altra parte non essere legato lo studio delle scritture d'apparato adoperate nei libri, manoscritti prima, a stampa poi, a scopo indicativo, designativo, esplicativo, per titolazioni, evidenziazione di parti di testo, guida alla lettura ecc.; studio attraverso il quale è possibile ricostruire l'itinerario di uso del testo predisposto ciascuna volta dai confezionatori del libro con strategie grafiche particolari, ognuna delle quali ha evidentemente alle spalle una storia di modelli, di influenze, di interpretazioni, che va puntualmente ricostruita ${ }^{4}$.

Per ultimo vorrei segnalare un altro fenomeno che meriterebbe ampie ricerche e particolare attenzione; quello, cioè, costituito dalla presenza nel basso medioevo o nel periodo rinascimentale di scriventi per conto di altri, evidentemente incapaci di scrivere per proprio conto, eppure costretti ad usare la scrittura per assolvere a precisi obblighi giuridici, amministrativi, fiscali e così via; questi scriventi delegati (corrispondenti agli $v \pi \sigma \gamma \rho \alpha$ (1) $\varepsilon i n$ dell'Egitto

${ }^{43}$ Cf. per questo tipo di prospettiva di ricerca L. MIGLIO, Considerazioni ed ipotesi sul libro «borghese» italiano del Trecento. A proposito di un'edizione critica dello «Specchio Umano» di Domenico Lenzi, in "Scrittura e Civiltà», 3 (1979), pp. 309-27; A. PETRUCCI, Il libro manoscrito, in Letteratura italiana, a cura di A. AsOR ROSA, II, Produzione e consumo, Torino 1983, pp. 499-524; per la produzione di «testi» cf. A. CICCHETTI-R. MORDENTI, La scrittura dei libri di famiglia, ibid., III, Le forme del testo. La prosa, 2, Torino 1984, pp. 1117-59; e, degli stessi autori, I libri di famiglia in Italia. I. Filologia e storiografia letteraria Roma 1985. Una ricerca particolare su un prolifico produttore di «scrittura» nel Quattrocento fiorentino: L. MIGLIO, L'avventura grafica di Iacopo Cocchi-Donati, funzionario mediceo e copista (1411-1479), in "Scrittura e Civiltà», 6 (1982), pp. 189-232.

${ }_{44}$ Cf. per questo A. PETRUCCI, Potere, spazi urbani e scritture esposte: proposte ed esempi, in Culture et idéologie dans la genèse de l'État moderne, Rome 1985, pp. 85-97; in particolare la parte seconda, pp. 143-169.

4s Cf. in generale A. PETRUCCI, La scrittura. Ideologia e rappresentazione, Torino 1986, (Piccola Biblioteca Einaudi, 473). In questo senso muovono anche alcune ricerche recenti di F. Troncarelli sulla tradizione dei testi di Boezio e Cassiodoro e sulla loro presentazione esterna; di lui si veda Tradizioni perdute. La "Consolalatio Philosophiae" nell'alto Medioevo, Padova 1981. 
greco-romano) ${ }^{* 6}$ appartenevano in genere all'ambiente medesimo degli analfabeti deleganti, di cui molto spesso erano parenti, compagni di lavoro, vicini di casa, e cui erano legati da un rapporto evidente di fiducia. In epoche di forte crescita dell'alfabetismo urbano e di esteso uso della scrittura gli scriventi delegati rappresentano una categoria socio-culturale di grande importanza, in quanto posta a confine fra l'area estremamente vasta dell' analfabetismo e della cultura orale e quella, ristretta, ma in espansione tumultuosa, della persone capaci di scrivere e di leggere ${ }^{47}$.

6. Una paleografia che si occupa prevalentemente dei problemi dell'uso e della funzione della scrittura e dei prodotti scritti nella società, e perciò stesso del ruolo sociale della scrittura, è indubbiamente assai diversa da quella tradizionale. Infatti, primo ed essenziale oggetto di quest'ultima è rimasto per molto tempo e rimane praticamente ancora la tipologia delle forme grafiche e la ricostruzione delle successioni di tali tipologie nel tempo, i passaggi insomma da sistema a sistema, dalla corsiva antica alla corsiva nuova, dalle minuscole precaroline alla carolina, dalla carolina alla gotica e così via; non a caso, del resto, la sua dimensione ideale è quella del manuale, funzionale a tale puntuale ricostruzione, forzatamente schematica (si pensi soltanto alla cosiddetta scrittura gotica!) di una realtà storica ben altrimenti frantumata e complessa. In realtà anche il tipo o modello di indagini paleografiche di cui qui si presentano le prospettive metodologiche e alcune almeno delle problematiche di fondo non è altro che storia della scrittura e delle testimonianze scritte. Ma storia non già del succedersi più o meno irreale in un tempo astratto di forme grafiche fra loro legate da presunte leggi evolutive individuate in modo più o meno meccàníco, bensì storia di singole situazioni di scrittura indagate in tutti $\mathrm{i}$ loro aspetti di uso, di pratiche, di abitudini, di tecniche, di cultura, di educazione, di sociabilità; una storia che comprende e abbraccia il fenomeno della scrittura, $\mathrm{e}$ perciò stesso dello scrivere, in tutta la sua estrema ricchezza e in tutta la sua complessità, e che studia gli uni accanto agli altri forme grafiche e prodotti scritti, scriventi e modi dello scrivere, funzione della scrittura nella società e cultura dello scritto ${ }^{48}$.

${ }^{4} \mathrm{H}$. C. YouTIE, ' $\gamma \pi 0 \gamma \rho \alpha \varphi \varepsilon u$ s. The social impact of illiteracy in Graeco-Roman Egypt, in "Zeitschrift für Papyrologie und Epigraphik», 17 (1975), pp. 202-21.

47 Cf. PETRUCCI, Per la storia, pp. 453-4; Id., Scrittura, alfabetismo ed educazione grafica, pp. 181-3.

48 Ritengo che le precisazioni qui addotte servano a configurare il proposto rinnovamento delle discipline paleograftehe come un allargamento del loro campo di indagine e non come un loro totale stravolgimento, o peggio come una fuoriuscita dal terreno della storia della scrittura; in effetti non comprendo per quali ragioni una storia globale della scrittura non debba e non possa essere anche, oltre che storia delle forme grafiche, anche storia dell'attività di scrittura, cioè dello scrivere, di cui le concrete realizzazioni grafiche (che si 
Scriveva Fernand Braudel nel lontano 1950: «Une histoire neuve n'est possible que par l'enorme mise à jour d'une documentation qui réponde à ces questions neuves"; e aggiungeva: "Je doute même que l'habituel travail artisanal de l'historien soit à la mesure de nos ambitions actuelles» ${ }^{49}$. In effetti il paleografo che intenda studiare nel concreto singole «situazioni di scrittura» e ricostruirle nella loro piena recchezza di testimonianza storica socio-culturale, dovrà, almeno in alcuni casi, rivolgersi a documentazioni di natura diversa rispetto a quelle finora abituali agli studi paleografici ed elaborare nuove metodologie d'approccio e d'esame, affinare quelle tradizionali, approfondire la propria preparazione professionale, allargare la sua personale esperienza a terreni finora mai esplorati ${ }^{\text {so. }}$.

Ma non per questo dimenticare (o peggio, non apprendere) l'enorme e fecondo tesoro costituito da più di tre secoli di studi, di indagini, di conoscenze, di acquisizioni; non per questo tagliare i rapporti con la tradizione disciplinare e impoverire con ciò stesso il suo lavoro, svilire le sue stesse conquiste. Mi sia consentito a tal proposito di chiudere il mio contributo con il monito autorevole che un grande, esperto ed acuto filologo classico, Sebastiano Timpanaro, rivolgeva recentemente ai suoi colleghi, ma che tutti i paleografi, e soprattutto i più giovani $\mathrm{e} i$ più arditi fra noi, dovrebbero attentamente meditare: «Tutte le volte che negli studi di antichità si fanno valere esigenze di rinnovamento, tanto più è necessario, se non si vuole costruire sulla sabbia, mantenere l'esercizio del «mestiere» e non perdere del tutto (pur non rimanendovi attaccati) quel tanto di gusto per il particolare che caratterizza il filologo nel senso «hermanniano» del termine e che non è nè mera pedanteria, nè mera

vorrebbero configurare come unico oggetto della disciplina) sono pur sempre diretti prodotti; cf. per questo le esplicite affermazioni di A. PRATESI, Paleografia in crisi?, in «Scrittura e Civiltà», 2 (1978), pp. 329-37, in particolare p. 331.

19 F. BRAUDEL, Positions de l'bistoire en 1950 (leģon inaugurale au Collège de France, $1^{\text {"r }}$ décembre 1950), in Id., Écrits sur l'bistoire, Paris 1984, p., 25.

51) Già nel 1978 Bartoli Langeli preconizzava, in una visione decisamente traumatica, una storia della scrittura che travolgesse «i consolidati confini, anche e anzitutto cronologici, e il compatto repertorio strumentale, conoscitivo, lessicale» della paleografia (Ancora su Paleografia e storia della scrittura, cit., p. 281), dando così origine alle prese di posizione, per loro parte altrettanto polemicamente decise, di A. Pratesi, di cui alla nota núm. 48. 
tecnica, ma esigenza di capire... L'unità vichiana di «filosofia» e di «filologia» viene menomata se è trascurato il momento specificamente filologico ${ }^{\mathrm{sl}}$.

\author{
ARMANDO PETRUCCI \\ Scuola Normale Superiore \\ (Pisa, Italia)
}

\begin{abstract}
RÉSUMÉ
L'auteur trace ici un tableau des études qui, tout au long de ces quarante dernières années, ont reconsidéré, sous differents angles, et en main d'experts de différentes formations, la relation entre l'écriture et les structures sociales. Dirigées, en principe, par des linguistes tel que $\mathbf{M}$. Cohen et par des experts de formation et d'influence marxiste originaires de pays d'Europe de l'Est, ces idées ont donné vie, d'une part, à une plus ample série d'études historiques de l'alphabétisme et, d'autre part, à des thèses proprement paléographiques destinées à étudier la fonction de l'écrit et de l'écriture dans les sociétés anciennes et médiévales.
\end{abstract}

\title{
SUMMARY
}

The author gives a scoop of the studies, led during the last forty years, by all kinds of experts, and from different points of view, on the relation between writing and social structure. Directed at the beginning by linguists such as $\mathbf{M}$. Cohen and by East European experts who follow the marxist trend, these ideas have given birth on the one hand to a wide range of historical studies about elimination of illiteracy and, on the other hand, to strictly palaeographic theses to study the function of the document and of writing for ancient and medieval societies.

"S. Timpanaro, Prefazione a Id., Contributi di filologia e storia della lingua latina, Roma 1978, p. 11. 